\title{
Bioévaluation De La Qualité Des Eaux Du Cours D'eau Menoua En Zone Périurbaine De Dschang, Ouest Cameroun
}

\author{
Ngameni Tchamadeu Norbert \\ Patricia Bi Asanga Fai \\ Mpoame Mbida
}

Laboratoire de Biologie et d'Ecologie Appliquées, Département de Biologie

Animale, Faculté des Sciences, Université de Dschang, Cameroun

\section{Kenko NKontcheu Daniel Brice}

Zoology Laboratory, Department of Zoology and Animal Physiology, Faculty of Science, University of Buea, Cameroon

doi: 10.19044/esj.2017.v13n27p368 URL:http://dx.doi.org/10.19044/esj.2017.v13n27p368

\begin{abstract}
We conducted a study to assess the impact of peri-urban agricultural activities of the city of Dschang on the water quality and macroinvertebrate structure of the Menoua stream. This study covered 3 stations (one reference station at the upstream of the peri-urban agricultural activities and two downstream) with a monthly sampling frequency from March 2015 to February 2016. The physico-chemical analysis were carried out according to standard protocols while the benthic macro-fauna was harvested using a haze net of $30 \mathrm{~cm}$ side and $400 \mu \mathrm{m}$ mesh size. Physico-chemical parameters such as temperature, $\mathrm{pH}$, conductivity, TDS (Total Dissolved Solids), salinity, turbidity, colour, SS (Suspended Solids), nitrate, ammonium and orthophosphate were analyzed. The inventory of benthic macroinvertebrates revealed a taxonomic richness of 42 families and 77 taxa and a gross abundance of 1833 individuals. Spatio-temporal monitoring of physicochemical and biological parameters provided the image of relatively low pollution. Metrics such as total taxonomic richness, richness of ETO (Ephemeroptera-Trichoptera-Odonata), relative abundance of Ephemeroptera-Trichoptera-Odonate (\% ETO), diversity of Shannon and Weaver and Pielou's equitability were determined at each of the three stations. These indices, unlike several other studies, increased downstream of anthropogenic activities. Low agricultural activity in the watershed has resulted in habitat diversification and availability of resources for benthic
\end{abstract}


macroinvertebrates, resulting in increased abundance and diversity.

Keywords: Agricultural, peri-urban, physic-chemical, benthic macroinvertebrates, pollution, watercourse, Dschang

\section{Résumé}

Nous avons réalisé une étude afin d'évaluer l'impact de l'activité agricole périurbaine de la ville de Dschang sur la qualité de 1'eau et la structure de peuplement des macroinvertébrés benthiques du cours d'eau Menoua. Cette étude a porté sur 3 stations (une station de référence en amont des activités agricoles périurbaine et deux autres en aval) avec une fréquence d'échantillonnage mensuelle de mars 2015 à février 2016. Les analyses physicochimiques ont été effectuées suivant les méthodes standards, tandis que la macrofaune benthique a été récoltée à l'aide d'un filet troubleau de $30 \mathrm{~cm}$ de côté et $400 \mu \mathrm{m}$ d'ouverture de maille. Les paramètres physicochimiques tels que la température, le $\mathrm{pH}$, la conductivité, le TDS (solides Totaux Dissous), la salinité, la turbidité, la couleur, les MES (Matières En Suspension), le nitrate, l'ammonium et l'orthophosphate ont été enregistrés. L'inventaire des macroinvertébrés benthiques révèle une richesse taxonomique de 42 familles et 77 taxons et une abondance brute de 1833 individus. Le suivi spatio-temporel des paramètres physico-chimiques et biologique a fourni l'image d'une pollution relativement faible. Les métriques tels que la richesse taxonomique totale, la richesse des ETO (Ephéméroptère-Trichoptère-Odonate), l'abondance relative des Ephéméroptères-Trichoptère-Odonate (\%ETO), la diversité de Shannon et Weaver et l'équitabilité de Pielou ont été déterminés au niveau de chacune des 3 stations. Ces indices contrairement à plusieurs autres études augmentent en aval des activités anthropiques. La faible activité agricole dans le bassin versant a entrainé la diversification d'habitats et la disponibilité de ressources pour les macroinvertébrés benthiques entrainant par conséquent une augmentation de leur abondance et de leur diversité.

Mots-clés : agricoles, périurbaines, physico-chimique, macroinvertébrés benthiques, pollution, cours d'eau, Dschang

\section{Introduction}

Dans les pays d'Afrique francophone, les grandes villes connaissent des taux de croissance de l'ordre de $7 \%$ par an en moyenne, ce qui signifie qu'en 2020 la population urbaine pourrait représenter environ $60 \%$ de la population totale (Margiotta, 1997). Ce flux migratoire est à l'origine de nombreux problèmes socio-économiques (taux de chômage élevé et insécurité alimentaire) et environnementaux (problème de gestion des 
ressources en eau, des déchets et de l'assainissement) (Margiotta, 1997; Kouam et al., 2010). A Dschang où la présente étude a été réalisée, la forte croissance démographique avec une population qui est passée de 49147 habitants en 1992 à 109576 en 2006 (INS, 2007) a conduit un bon nombre de populations à s'intéresser à l'agriculture périurbaine pour résoudre le problème de chômage, de sous-alimentation et des carences nutritionnelles. L'agriculture périurbaine joue donc un rôle clé dans l'approvisionnement des villes. Les faibles coûts de commercialisation, la proximité des marchés de consommation, la réduction du chômage sont les principaux aspects positifs qui ont facilité le développement de l'agriculture périurbaine. Ces avantages impliquent son maintien dans le temps, surtout dans les situations où le transport vers les zones rurales est difficile. Bien que son utilité soit reconnue, l'agriculture périurbaine exerce une pression sur les ressources en eau. Différents travaux se sont intéressés à l'impact de l'agriculture périurbaine sur la qualité des eaux (Wade, 2003; Cissé et al., 2003). En effet, l'intensification agricole reste l'une des causes majeures de dégradation des systèmes aquatiques (Leight et al., 2010; Sass et al., 2010). Les contaminants issus des intrants agricoles jouent sur le développement du biote présent. Les perturbations des écosystèmes aquatiques se ressentent très souvent au niveau des communautés biologiques (Moisan et Pelletier, 2008). Ainsi, la surveillance de l'intégrité de ces écosystèmes s'est beaucoup basée aujourd'hui sur des mesures relatives aux communautés biologiques en place, notamment les macroinvertébrés. Ces organismes diversement répandus au niveau des différents strates de l'eau se caractérisent par leur polluo-sensibilité différentielle; caractère utilisé en bioindication des écosystèmes aquatiques. Le suivi environnemental basé sur les macroinvertébrés est très efficace parce que ceux-ci ne répondent pas seulement aux polluants, mais aussi aux changements dans l'utilisation des terres dans le bassin versant et aux changements physiques et biologiques de 1'habitat, qui sont difficiles à évaluer avec un suivi classique de toxicologie ou de chimie (Rosenberg et Resh, 1993). Au Cameroun, les macroinvertébrés benthiques ont été utilisés pour évaluer l'intégrité écologique des milieux d'eau douce en zone urbaine et périurbaine (Tchakonté, 2016; Foto et al., 2012; Foto et al., 2013; Ajeagah, 2014). Cependant, leur application comme bioindicateurs pour évaluer la qualité des eaux des cours d'eau en zone agricole périurbaine est encore peu développée.

Notre étude s'est focalisée sur le cours d'eau Menoua au quartier Tsinbing à l'entrée de la ville de Dschang. Son bassin versant est cultivé, principalement à des fins vivrières avec presque les mêmes cultures en saison sèche qu'en saison des pluies. L'exploitation de cet espace périurbain pour la culture vivrière est peu à peu occupée par les mini-cités et les maisons d'habitation depuis la création d'une université à cycle complet dans 
la ville en 1993. Il se pose donc un problème de terre, la pratique de la jachère devient presque impossible et les parcelles disponibles sont exploitées en continu. Or, sur le plan écologique, la réduction des jachères à cause de la diminution des surfaces disponibles entraine la baisse de la fertilité des sols et l'utilisation des engrais reste le principal moyen de fertilisation des ces derniers. Aussi, l'absence de jachère augmente la pression des ennemies des cultures tels les parasites et les ravageurs ce qui pousse les agricultures à utiliser les pesticides chimiques (Temgoua et al., 2012). Cette pratique peut avoir des conséquences néfastes sur la qualité des eaux du cours d'eau environnant.

Ce travail réalisé pour la première fois sur un hydrosystème traversant la zone agricole périurbaine de Dschang avait pour but d'évaluer l'impact de cette activité sur la qualité des ressources en eau et ce à travers une caractérisation physico-chimique et biologique.

\section{Méthodologie}

\section{Zone d'étude et station d'échantillonnage}

La ville de Dschang constitue notre région d'étude. Elle est une subdivision administrative du Département de la Menoua, Région de l'Ouest-Cameroun. Elle appartient au grand ensemble géographique des Hautes terres de l'ouest et est située sur le versant sud des Monts Bamboutos. Elle est comprise entre $5^{\circ} 25^{\prime}-5^{\circ} 30^{\prime}$ de Latitude Nord et $10^{\circ}-10^{\circ} 5^{\prime}$ de Longitude Est. La zone urbaine occupe essentiellement un ensemble de collines. Le climat de la région de Dschang est de type équatorial de type camerounien, fortement influencé par des hautes altitudes. Certains auteurs lui ont attribué l'appellation de climat camerounien montagnard ou climat camerounien d'altitude. L'analyse des données de la station météorologique de Dschang de 2001 à 2009 montre qu'il existe deux saisons : une longue saison de pluie allant de mars à octobre $(8$ mois) et une courte saison sèche s'étalant sur 4 mois (de novembre à février). La période la plus arrosée est comprise entre le mois de juin $(243,4 \mathrm{~mm})$ et le mois de septembre $(235.2$ $\mathrm{mm})$. Le maximum de précipitations est enregistré au mois d'Août $(313,9$ $\mathrm{mm})$ et le minimum en décembre $(9,5 \mathrm{~mm})$. La température moyenne annuelle est de $21^{\circ} \mathrm{C}$. La durée de l'ensoleillement de 8,5 heures à 2 heures par jour respectivement en saison sèche et en saison pluvieuse, et conditionne les températures journalières. L'air est perpétuellement frais et tend à sa saturation au petit matin, d'où la présence régulière du brouillard ou de la brume dans l'atmosphère avant le levé du soleil. La figure 1 représente la localisation de la ville de Dschang. 


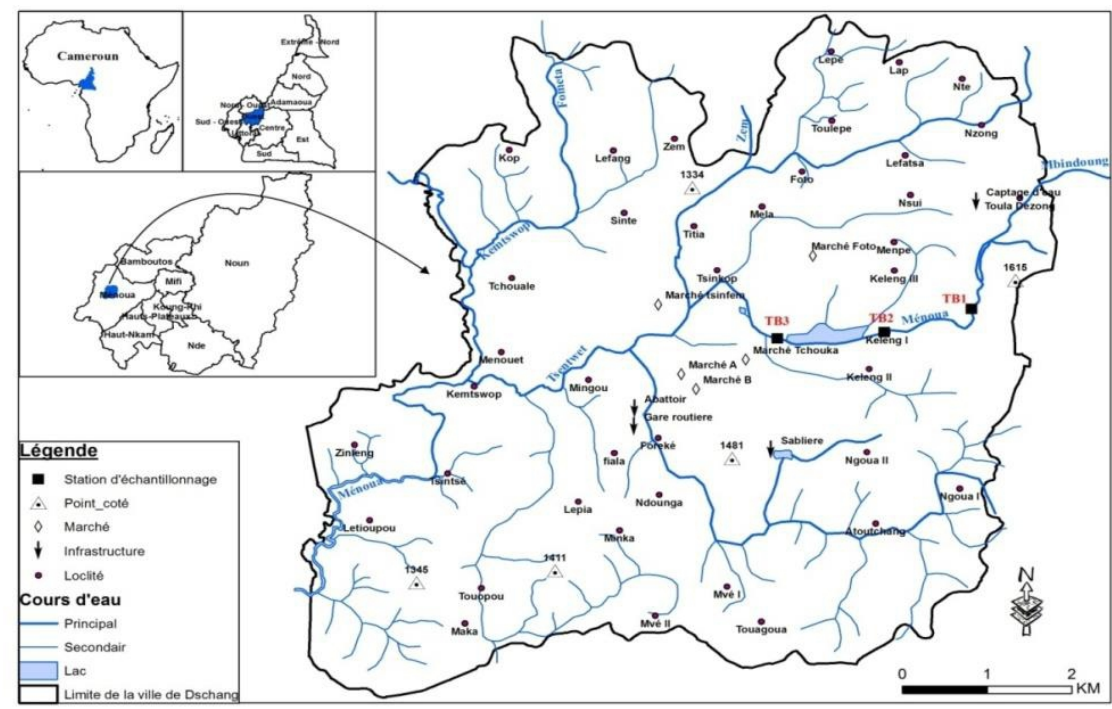

Figure 1: Carte de situation de la zone d'étude et de localisation des stations de prélèvement Stations d'étude

Compte tenu de l'objectif général de l'étude à savoir la caractérisation physico-chimique et biologique du cours d'eau Menoua traversant la zone agricole périurbaine de la ville de Dschang, trois stations sont retenues sur ce cours d'eau au quartier Tsinbing :

La station TB1 $\left(05^{\circ} 27^{\prime} 48.3^{\prime \prime} \mathrm{N}, 010^{\circ} 05^{\prime} 18.2^{\prime}\right.$ ' E) : cette station est située à l'entrée nord de la ville de Dschang au lieu dit "Château », à environs $2 \mathrm{~km}$ de la gare routière. Sa distance à la source est faible et son environnement est constitué sur la rive gauche et droite des plantes de raphias parsemées d'arbustes. Ombragée, son lit présente un substrat hétérogène constitué de sable et de gravier, mais aussi de la matière organique en décomposition (débris de feuilles, branches...). Cette station est située loin des habitations et sans aménagement, en amont du barrage d'alimentation en eau potable de la ville de Dschang. Elle a servi de référence car elle est proche de la source et peu touchée par les nuisances anthropogéniques.

La station $\mathbf{T B 2}\left(05^{\circ} 27^{\prime} 02.1^{\prime \prime} \mathrm{N}, 010^{\circ} 04^{\prime} 36.5^{\prime}\right.$ ' E) : cette station est localisée dans une zone de culture qui se transforme peu à peu en zone d'habitation, à environs $2 \mathrm{~km}$ de la station TB1. Le cours d'eau avant d'arriver dans cette station traverse une zone de grandes influences agricoles. Les activités agricoles sont pratiquées sur l'ensemble du bassin versant. La végétation sur les deux rives est dominée par les cultures vivrières. Le fond est constitué de sable et d'argile. Cette station se trouve à $200 \mathrm{~m}$ en amont du lac municipal de Dschang, et à $100 \mathrm{~m}$ en aval d'une zone de lessive. 
Cette position a permis d'avoir l'influence des activités agricoles afin de juger la qualité de l'eau avant son entrée dans le lac.

La station $\mathbf{T B 3}\left(05^{\circ} 26^{\prime} 57.7^{\prime}\right.$ ' $\mathrm{N}, 010^{\circ} 03^{\prime} 51.2^{\prime}$ ' E) : Situé seulement à $100 \mathrm{~m}$ en aval du lac municipal de Dschang, elle est directement sous son influence. Cette station a permis de juger la qualité de l'eau qui sort du lac. La végétation bordante est dominée par Tithonia diversifolius. Le substrat est formé de gravier et de sable. Cette station se trouve à $300 \mathrm{~m}$ en amont de la confluence du cours d'eau étudié avec son affluent chargé des lixiviats et déchets en provenance des campus et des activités autour de l'université de Dschang.

\section{Mesure des paramètres hydromorphométriques}

Les mesures des paramètres hydromorphométriques (débit, vitesse moyenne, section mouille, largeur moyenne, profondeur moyenne) ont été effectuées sur le terrain pour chaque mois et pour chaque station pendant toute la période de l'étude.

L'estimation de la vitesse du courant a été effectuée en surface du cours axial à l'aide d'un flotteur et d'un chronomètre. Le flotteur est lâché en dérive sur une distance $(\mathrm{d})$ connue. Le temps $(\mathrm{t})$ mis par le flotteur à parcourir cette distance a permis de calculer la vitesse par la formule suivante :

$\mathrm{V}=\mathrm{d} / \mathrm{t}(\mathrm{m} / \mathrm{s})$

L'estimation de la section mouillée s'est faite par la mesure de la largeur moyenne (L) et de la profondeur moyenne (P). La section a été approximativement calculée de la façon suivante :

$\mathrm{S}=\mathrm{L} \times \mathrm{P}$

Le débit a été donc calculé par la formule :

$\mathrm{Q}=\mathrm{S} \times \mathrm{V}\left(\mathrm{m}^{3} / \mathrm{s}\right)$ où $\mathrm{S}$ est la section mouillée et $\mathrm{V}$ la vitesse moyenne

\section{Mesures des paramètres physicochimiques}

Les mesures des paramètres physicochimiques se sont déroulées à la fois sur le terrain et au laboratoire suivant les recommandations de APHA (2009) et Rodier et al. (2009). Quatre paramètres physico-chimiques ont été enregistrés sur le terrain: la température, le $\mathrm{pH}$, la salinité, le TDS et la conductivité ont été mesurées par une sonde multiparamètre modèle waterproof IP57.

Pour les paramètres à mesurer au laboratoire (turbidité, nitrate, ammonium, orthophosphates, MES, couleur) les échantillons d'eau ont été prélevés dans des flacons en polyéthylène de $500 \mathrm{ml}$, à double bouchage préalablement rincés avec les eaux du milieu, puis conservés en enceinte réfrigérée dans une glacière contenant des carboglaces. Ils ont été immédiatement transportés au laboratoire de Botanique Appliquée de 
l'Université de Dschang et gardés au frigo avant les analyses dans les 24 heures suivant la récolte selon les techniques et méthodes préconisées par Rodier et al. (2009).

\section{Echantillonnage des macroinvertébrés benthiques}

Les prélèvements ont été réalisés de mars 2015 à février $2016(12$ mois), avec une fréquence d'échantillonnage mensuelle. L'échantillonnage a été effectué à l'aide d'un troubleau constitué d'un filet conique de $400 \mu \mathrm{m}$ de vide de maille et $50 \mathrm{~cm}$ de profondeur fixé sur un cadre métallique de 30 $\mathrm{cm}$ de côté, monté sur une manche en acier de $150 \mathrm{~cm}$ de long. Il s'est fait suivant l'approche multihabitat proposée par Stark et al. (2001), adaptée de Babour et al. (1999), qui consiste à effectuer dans chaque station, à chaque campagne, un total de 20 coups de filet dans différents micro-habitats caractérisés par le couple substrat/vitesse. Cette méthode consiste à déposer le cadre du troubleau à même le substrat et balayer le fond de l'eau par des mouvements de va et vient sur une distance de $50 \mathrm{~cm}$. Les organismes ainsi récoltés sont fixés au formol à $10 \%$ sur le terrain. Au laboratoire, les spécimens sont rincés sur un tamis de $400 \mu \mathrm{m}$ d'ouverture de maille puis conservés dans de l'alcool à $70^{\circ}$.

La totalité des spécimens a été identifiée le plus précisément possible, sous un stéréoscope modèle discovery V8, Zeiss, ceci à l'aide des clés de détermination. Parmi les clés utilisées, celles de Durand et Lévêque (1981) et Tachet et al. (2010) traitent des invertébrés de façon générale. Les autres clés traitent spécialement des insectes (Dejoux et al., 1981 ; Diomandé et al., 2000 ; Day et al., 2002 ; De Moor et al., 2003 ; Stals \& De Moor, 2007), des crustacés (Day et al., 2001), des mollusques (Brown, 1994 ; De Moor \& Day, 2002).

\section{Analyse de la structure du peuplement}

La structure du peuplement des macroinvertébrés benthiques dans chaque station a été étudiée à l'aide des métriques tels que : la richesse taxonomique totale, la richesse des Diptères, la richesse des ETO (Ephéméroptère-Trichoptère-Odonate), la richesse des Insectes, l'abondance totale, l'abondance relative des Ephéméroptères-Trichoptère-Odonate (\%ETO) et l'abondance relative des Insectes. Les indices tels que la diversité de Shannon et Weaver et l'équitabilité de Pielou ont été également calculés dans chaque station afin d'évaluer l'impact d'une éventuelle modification du milieu sur les macroinvertébrés benthiques.

Une analyse des variances (ANOVA à un facteur) a été réalisée afin de mettre en évidence les variations des paramètres physico-chimiques entre les différentes stations d'échantillonnage. De plus, le test de corrélations de Pearson a été utilisé pour établir une relation entre variables 
physico-chimiques et biologique. Les logiciels Microsoft Excel 2007 et SPSS version 9.0 ont été utilisés pour le traitement des données.

\section{Résultats}

\section{Variables hydromorphométriques}

Les valeurs des paramètres hydromorphométriques obtenues des trois stations d'étude sont représentés dans le tableau ci-dessous (tableau 1).

Tableau 1: Valeurs moyennes et écart-types des paramètres hydromorphologiques mesurés au niveau des 3 stations d'échantillonnage (TB1, TB2, TB3) pendant la période d'étude

\begin{tabular}{ccccc}
\hline \multirow{2}{*}{ Stations } & \multicolumn{4}{c}{ Paramètres } \\
\cline { 2 - 5 } & Profondeur $(\mathrm{m})$ & Largeur $(\mathrm{m})$ & Vitesse $(\mathrm{m} / \mathrm{s})$ & Débit $\left(\mathrm{m}^{3} / \mathrm{s}\right)$ \\
\hline TB1 & $0,43 \pm 0,12$ & $3,51 \pm 0,77$ & $0,69 \pm 0,21$ & $1,20 \pm 0,81$ \\
TB2 & $0,71 \pm 0,22$ & $2,96 \pm 0,73$ & $0,46 \pm 0,15$ & $1,14 \pm 0,86$ \\
TB3 & $0,51 \pm 0,16$ & $4,07 \pm 1,55$ & $0,69 \pm 0,25$ & $1,72 \pm 1,39$ \\
\hline
\end{tabular}

Il en ressort que la station TB1 est la moins profonde de toutes avec une profondeur moyenne de $0,43 \pm 0,12 \mathrm{~m}$. La station TB2 est la plus profonde avec une hauteur moyenne estimée à $0,71 \pm 0,22 \mathrm{~m}$. Avec une largeur moyenne de 4,07 $\pm 1,55 \mathrm{~m}$, la station TB3 est la plus large. La station TB2 est la moins large. Les valeurs les plus élevées de la vitesse ont été enrégistrées en amont au niveau de la station TB1 $(0,69 \pm 0,21 \mathrm{~m} / \mathrm{s})$ et en aval au niveau de la station TB3 $(0,69 \pm 0,25 \mathrm{~m} / \mathrm{s})$. La vitesse moyenne la plus faible a été enrégistrée au niveau de la station TB2 $(0,46 \pm 0,15 \mathrm{~m} / \mathrm{s})$. En ce qui concerne le débit, elle a présenté peu de variabilités d'une station à l'autre avec des valeurs allant de $1,14 \pm 0,86 \mathrm{~m}^{3} / \mathrm{s}$ (TB2) à $1,72 \pm 1,39 \mathrm{~m}^{3} / \mathrm{s}$ (TB3).

\section{Paramètres physico-chimiques}

Les variations spatiales et mensuelles des valeurs des 11 paramètres physico-chimiques mesurés sont représentées par la figure 2 .

Le $\mathrm{pH}$ se situe entre 6,4 au mois de février à la station TB3 et 8,36 au mois de juin à la station TB1(Figure 2A). Les plus faibles valeurs de $\mathrm{pH}$ sont enrégistrées au mois de février au niveau de toutes les stations. Au niveau de chaque station, les moyennes de $\mathrm{pH}$ indiquent de manière générale une tendance à une légère alcalinité. Le test ANOVA ne montre aucune différence significative $(p>0,05)$ entre les valeurs obtenues au niveau des 3 stations.

Durant toute la période d'étude, la température obtenue à la station TB3 est significativement $(\mathrm{p}<0,05)$ plus élevée que celles enregistrées aux stations TB1 et TB2. a peu varié (Figure $2 \mathrm{~B})$. La valeur la plus faible $\left(17^{\circ} \mathrm{C}\right)$ 
a été enrégistrée à la station TB2 au mois de Novembre. La valeur la plus forte de $24,4^{\circ} \mathrm{C}$ a été obsevée à la station TB3 au mois de février. D'une manière générale, le mois de février n'a enrégistrée que des valeurs élevées. La température suit un gradient croissant de l'amont (station TB1) vers l'aval (station TB3) de décembre à Juillet. Au mois de Novembre, elle a plutôt subit une baisse à la station TB2 comparée à la station TB1.

Contrairement à la température, la conductivité a présenté des valeurs assez variées d'une station à l'autre et d'un mois à l'autre (Figure 2C). La valeur minimale $(32 \mu \mathrm{S} / \mathrm{cm})$ a été mésurée aux mois de septembre et février au niveau des stations TB1 et TB2 respectivement. La plus grande valeur de la conductivité $(74 \mu \mathrm{S} / \mathrm{cm})$ a été enrégistrée au niveau de la station TB3 au mois de novembre. Comme la température, la conductivité croit significativement $(\mathrm{p}<0,05)$ de la station TB1 à la station TB3. Il en est de même pour les valeurs de TDS qui ont suivies un gradient signifiactivement $(\mathrm{p}<0,05)$ croissant de l'amont (station TB1) vers l'aval (station TB3) durant toute la période d'étude (Figure $2 \mathrm{E})$. Le pic $(40 \mathrm{mg} / \mathrm{l})$ et la plus faible valeur (18mg/l) ont été enrégistrés au mois de février au niveau des stations TB3 et TB2 respectivement.

Pendant toute la période de l'étude, les valeurs de la salinité ont significativement $(\mathrm{p}<0,05)$ varié entre la station TB3 et les deux autres stations (Figure 2D). Elles s'échelonnent entre $14 \mathrm{mg} / \mathrm{l}$ et $32 \mathrm{mg} / \mathrm{l}$ respectivement aux mois de novembre et février aux stations TB1 et TB2 et au mois de novembre à la station TB3.

Les teneurs en nitrates ont été relativement faibles durant toute la période de l'étude, excepté le pic observé $(2,4 \mathrm{mg} / \mathrm{l})$ au niveau de la station TB2 au mois d'octobre (Figure $2 \mathrm{~F}$ ). La valeur la plus faible $(0,0 \mathrm{mg} / \mathrm{l})$ a été enrégistrée à la station TB1 au mois de septembre. Les variations de ce paramètre ne sont pas significatives $(p>0,05)$ entre les 3 stations.

L'azote ammoniacal a été retrouvé dans les eaux sous forme de trace entre le mois de mars et le mois d'août au niveau des trois stations (Figure $2 \mathrm{G}$ ). Les valeurs relativement élevées ont été enrégistrées aux mois de septembre et octobre pour rechuter entre la mois de novembre à février. Les teneurs enrégistrées pendant toute la durée de cette étude ne sont pas significativement $(\mathrm{p}>0,05)$ différentes entre les 3 stations et sont comprises entre $0,0 \mathrm{mg} / 1$ au mois d'août en TB3 et $0,28 \mathrm{mg} / 1$ au mois d'octobre en TB2.

Les valeurs de la turbidité observées au cours de cette étude ne varient pas significativement $(\mathrm{p}>0,05)$ entre les 3 stations. Elles ont varié de 0,0 NTU (aux stations TB1 et TB2 au mois d'avril) à 22 NTU au mois d'août à la station TB2 (Figure 2H). Les valeurs les plus élevées ont été observée entre le mois de mai et le mois de septembre dans toutes les stations. Les profils d'évolution sont très irréguliers et relativement similaires au niveau des trois stations. 
Les MES présentent au titre même que la turbidité des valeurs plus élevées entre le mois de mai et le mois de septembre dans toutes les stations (Figure 2I). La valeur la plus élevée $(30 \mathrm{mg} / \mathrm{l})$ a été observée à la station TB3 au mois de juillet et la plus basse $(1 \mathrm{mg} / \mathrm{l})$ à la station TB1 au mois de février. Le mois de juillet a enrégistré les valeurs les plus élevées dans chacune des 3 stations. Ce paramètre ne diffère pas significativement ( $p>$ 0,05 ) d'une station à l'autre.

Les valeurs de la couleur ne varient pas significativement $(p>0,05)$ entre les 3 stations. Elles s'échélonnent entre 0 à 72 Pt.Co pendant toute la période d'étude (Figure J). Le mois de février a présenté la plus faible valeur dans toute les stations. Le mois de janvier a également enrégistré des faibles valeurs. La valeur la plus élevée a été enrégistrée au mois de juillet à la station TB3. Le mois d'août a présenté des valeurs élevées aux stations TB1 et TB2.

Pendant toute la période de l'étude, les teneurs de l'orthophosphate n'ont pas significativement $(\mathrm{p}>0,05)$ varié d'une station à l'autre (Figure $2 \mathrm{~K})$. On note une augmentation croissante de la teneur en orthophosphate au niveau de la station TB3 au mois de Juin et une baisse au mois d'octobre au niveau des stations TB1 et TB2. Le mois de juin a enrégistrée les valeurs les plus élevées dans toutes les stations.
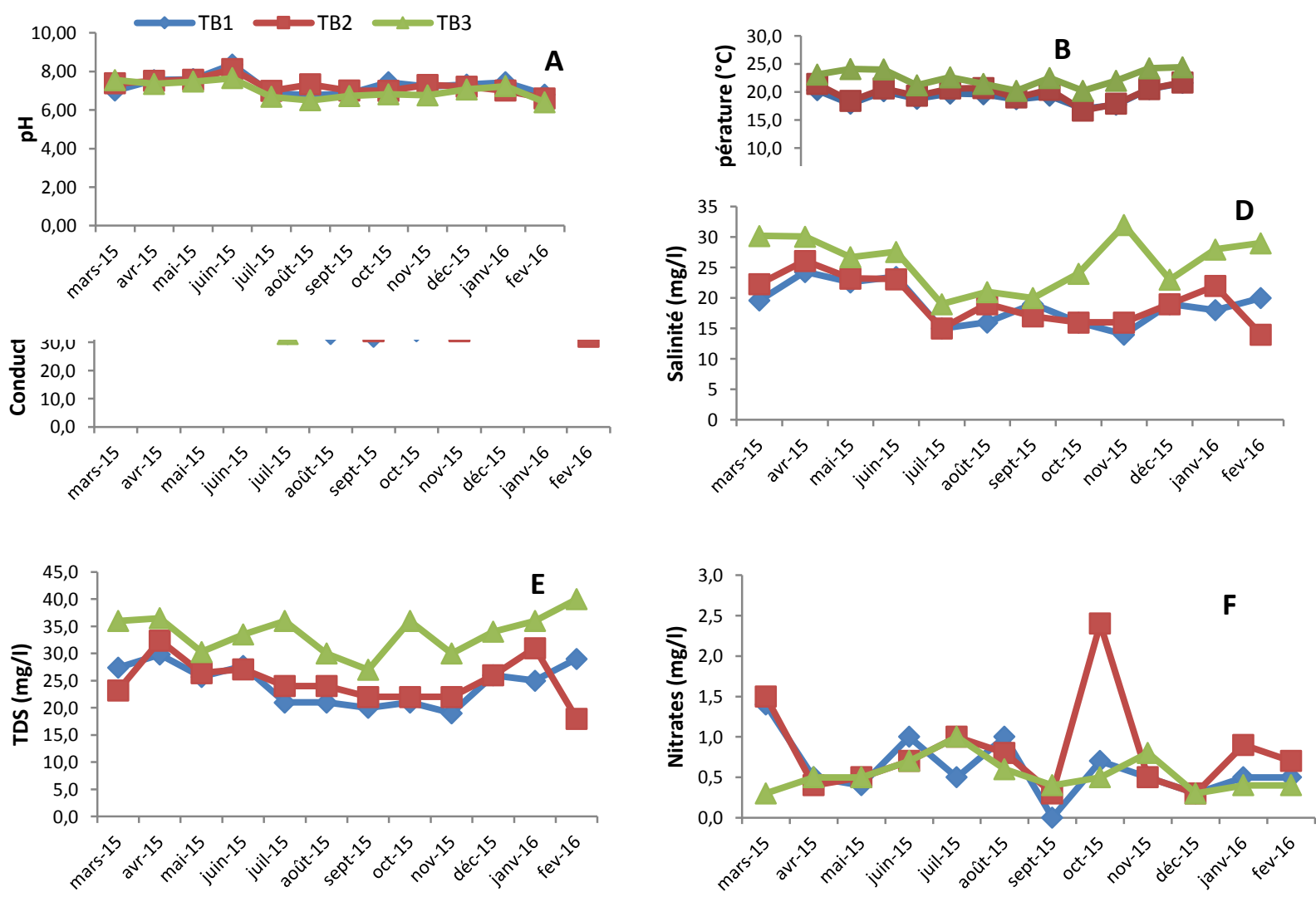

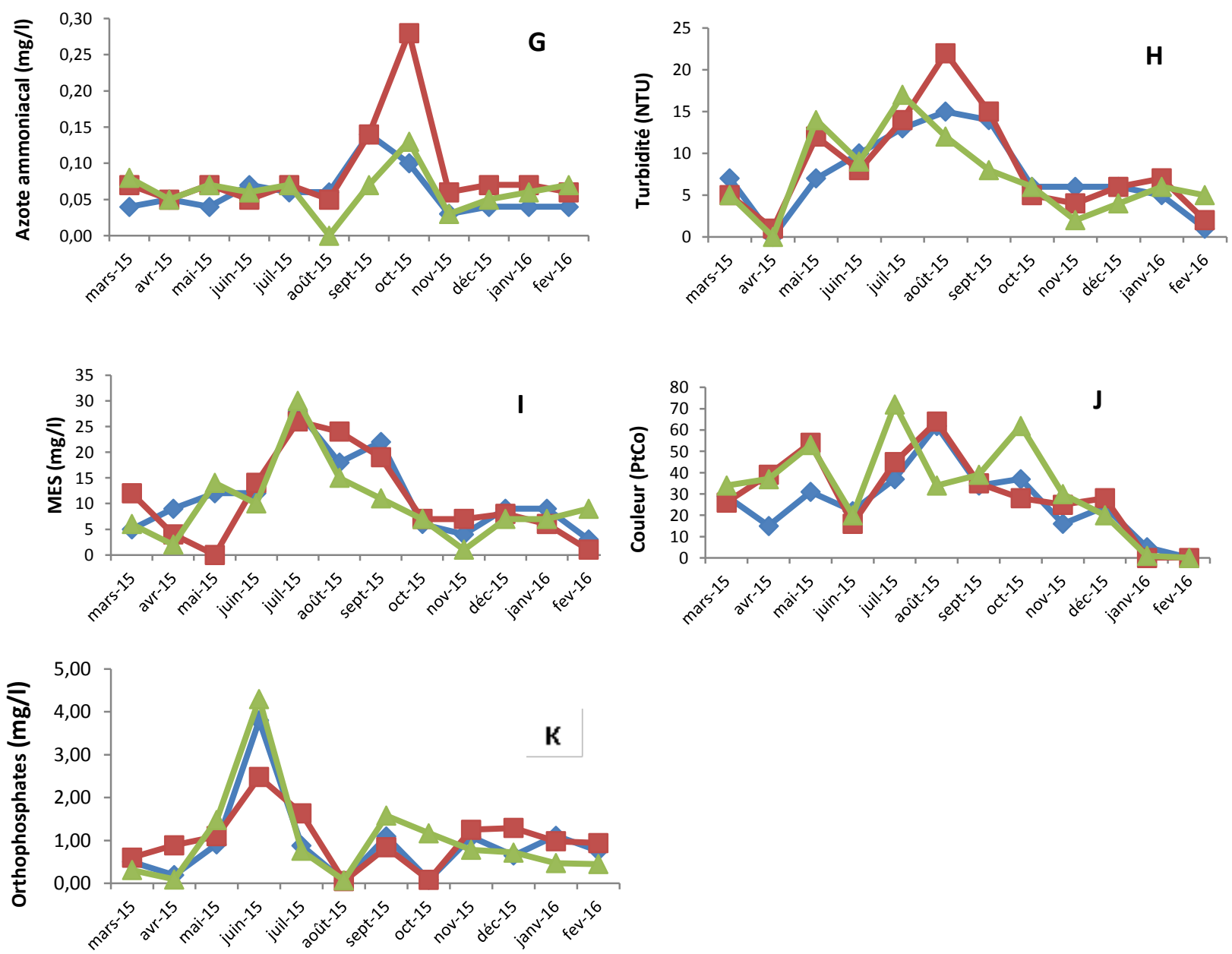

Figure 2 : Variations spatiales des valeurs mensuelles du $\mathrm{pH}$ (A), de la température (B), de la conductivité (C), du TDS (D), de la salinité (E), du nitrate (F), de l'azote ammoniacal $(\mathrm{G})$, des MES (H), de la turbidité (I), de la couleur (J) et de l'orthophosphate $(\mathrm{K})$

L'inventaire de la macrofaune benthique du cours d'eau périurbain de la ville de Dschang a permis de recenser 77 taxons d'invertébrés benthiques répartis en 42 familles (tableau 2). Les arthropodes sont le groupe le plus riche en taxons, représentant $88 \%$ de la totalité de la faune récoltée. Ils sont représentés par deux classes: Les insectes et les crustacés. Les insectes constitués de 7 ordres (Ephéméroptères $(9 \%)$, Trichoptères $(7 \%)$, Odonates $(41 \%)$, Hémiptères $(16 \%)$, Plécoptères $(1 \%)$, Coléoptères $(10 \%)$, Diptères $(12 \%)$ ) et de 33 familles dominent avec $84 \%$ des taxons. L'ordre des 
Odonates essentiellement dominé par la famille des Libellulidae (10 taxons) est le plus représenté en termes de familles (8 familles). Les crustacés quant à eux sont constitués d'une seule famille (Potamonidae) appartenant à l'ordre des Décapodes et d'un seul taxon (Potamonautes brincki). Les Plathelminthes constitués de la seule famille des Dendrocoelidae sont le groupe le moins représenté avec 1\%, suivis des Annélides et des mollusques avec 5\% chacun. Les annélides sont représentés par les familles des Glossiphoniidae, des Tubificidae et des Lumbricidae. Quant aux Mollusques, ils sont représentés par une seule famille (Planorbidae) appartenant à l'ordre des Gastéropodes et constitués de 4 taxons.

Au total, 1833 individus ont été récoltés au cours de cette étude. Les insectes qui comptent $91 \%$ des individus récoltés dominent le peuplement de macroinvertébrés. Ils sont représentés par 27\% d'odonates, 26\% deTrichoptères, $14 \%$ de Diptères, $10 \%$ de Coléoptères, $6 \%$ d'Hémiptères, $4 \%$ d'Ephéméroptères et $0,05 \%$ de Plécoptères. Les Mollusques ont suivis avec 122 individus soit une abondance relative de 6\%. L'abondance brute la plus élevée a été observée à la station TB3 (1118 spécimens, essentiellement constitué de Chironomidae) alors que la plus faible a été enrégistrée à la station TB1 (240 individus).

Tableau 2 : Liste des taxa de la macrofaune benthique récoltés au niveau des 3 stations d'échantillonnage ; $n d=$ taxon non déterminé

\begin{tabular}{|c|c|c|c|c|c|c|c|}
\hline $\begin{array}{c}\text { Embranche } \\
\text { ments }\end{array}$ & Classes & Ordres & Familles & $\begin{array}{c}\text { Genres ou } \\
\text { espèces }\end{array}$ & $\begin{array}{c}\text { TB } \\
1 \\
\end{array}$ & $\begin{array}{c}\text { TB } \\
2 \\
\end{array}$ & $\begin{array}{c}\text { TB } \\
3 \\
\end{array}$ \\
\hline \multirow[t]{14}{*}{ Arthropodes } & \multirow[t]{14}{*}{ Insectes } & \multirow[t]{5}{*}{$\begin{array}{l}\text { Ephémérop } \\
\text { tères }\end{array}$} & $\begin{array}{c}\text { Leptophebiida } \\
\text { e }\end{array}$ & $\begin{array}{l}\text { Habroleptoide } \\
\text { s sp. }\end{array}$ & 0 & 1 & 0 \\
\hline & & & Caenidae & Caenis sp. & 0 & 13 & 0 \\
\hline & & & Baetidae & Baetis sp. & 4 & 47 & 2 \\
\hline & & & Siphlonuridae & $\begin{array}{l}\text { Siphlonurus } \\
\text { sp. }\end{array}$ & 0 & 16 & 0 \\
\hline & & & Ephéméridae & Afromera sp. & 1 & 0 & 0 \\
\hline & & \multirow{5}{*}{$\begin{array}{c}\text { Trichoptère } \\
\text { s }\end{array}$} & Hydroptilidae & Stactobia sp. & 2 & 0 & 4 \\
\hline & & & Ecnomidae & Ecnomus sp. & 0 & 3 & 0 \\
\hline & & & \multirow{3}{*}{$\begin{array}{l}\text { Hydropsychid } \\
\text { ae }\end{array}$} & $\begin{array}{c}\text { Cheumatopsyc } \\
\text { he sp. }\end{array}$ & 5 & 13 & $\begin{array}{c}24 \\
8\end{array}$ \\
\hline & & & & $\begin{array}{c}\text { Hydropsyches } \\
\text { sp. }\end{array}$ & 4 & 25 & $\begin{array}{c}18 \\
1\end{array}$ \\
\hline & & & & $\begin{array}{c}\text { Cheumatopsyc } \\
\text { he lepida }\end{array}$ & 0 & 1 & 0 \\
\hline & & \multirow[b]{4}{*}{ Odonates } & \multirow[t]{2}{*}{$\begin{array}{c}\text { Calopterygida } \\
\mathrm{e}\end{array}$} & $\begin{array}{c}\text { Phaon } \\
\text { iridipennis }\end{array}$ & 4 & 10 & 4 \\
\hline & & & & Calopteryx sp. & 0 & 4 & 0 \\
\hline & & & \multirow[b]{2}{*}{ Corduliidae } & $\begin{array}{l}\text { Phyllomacrom } \\
\text { ia picta }\end{array}$ & 11 & 2 & 3 \\
\hline & & & & $\begin{array}{l}\text { Oxygastra } \\
\text { curtsii }\end{array}$ & 1 & 0 & 0 \\
\hline
\end{tabular}




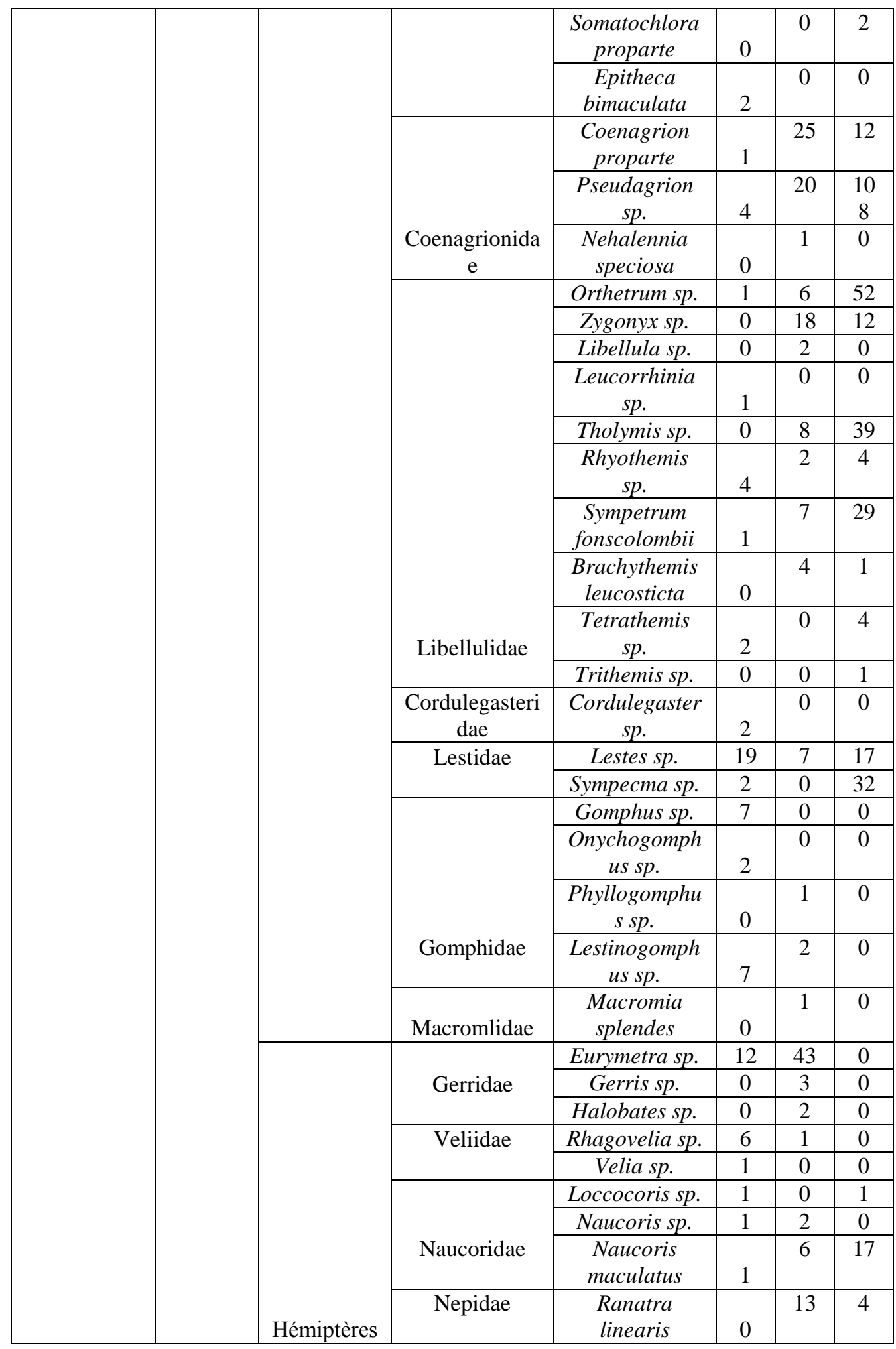




\begin{tabular}{|c|c|c|c|c|c|c|c|}
\hline & & & & Nepa sp. & 0 & 0 & 1 \\
\hline & & & $\begin{array}{c}\text { Hydrometrida } \\
\mathrm{e}\end{array}$ & $\begin{array}{c}\text { Hydrometra } \\
\text { sp. }\end{array}$ & 1 & 0 & 0 \\
\hline & & Plécoptères & Nemouridae & Nemoura sp. & 1 & 0 & 0 \\
\hline & & & & $\begin{array}{c}\text { Orectogyrus } \\
\text { specularis }\end{array}$ & 83 & 44 & 2 \\
\hline & & & & $\begin{array}{c}\text { Aulonogyrus } \\
\text { sp. }\end{array}$ & 29 & 20 & 0 \\
\hline & & & Gyrinidae & Gyrinus sp. & 2 & 1 & 0 \\
\hline & & & & Dineutus sp. & 0 & 4 & 0 \\
\hline & & & Elmidae & Limnius sp. & 0 & 11 & 0 \\
\hline & & $\begin{array}{c}\text { Coléoptère } \\
\text { S }\end{array}$ & Elmidae & $\begin{array}{c}\text { Potamophilus } \\
\text { sp. }\end{array}$ & 1 & 0 & 0 \\
\hline & & & Hydrophilidae & Enochrus sp. & 0 & 1 & 1 \\
\hline & & & & $\begin{array}{c}\text { Chironominae } \\
(S F)\end{array}$ & 0 & 5 & 9 \\
\hline & & & Chironomidae & $\begin{array}{c}\text { Chironomus } \\
\text { sp. }\end{array}$ & 0 & 67 & $\begin{array}{c}12 \\
0\end{array}$ \\
\hline & & & Tipulidae & Tipula sp. & 3 & 3 & 2 \\
\hline & & & Scatophagidae & $\begin{array}{l}\text { Acanthocnema } \\
\text { sp. }\end{array}$ & 0 & 0 & 1 \\
\hline & & & Simuliidae & $\begin{array}{c}\text { Prosimulium } \\
\text { sp. }\end{array}$ & 5 & 3 & 42 \\
\hline & & & & nd & 0 & 0 & 8 \\
\hline & & & Sciomyzidae & nd & 0 & 0 & 2 \\
\hline & & & Syrphidae & nd & 0 & 0 & 1 \\
\hline & & Diptères & Anthomyidae & $\begin{array}{c}\text { Limnophora } \\
\text { sp. }\end{array}$ & 0 & 0 & 2 \\
\hline & & Aranéides & // & // & 0 & 1 & 0 \\
\hline & & Acariens & $/ /$ & // & 0 & 3 & 0 \\
\hline & $\begin{array}{c}\text { Crustac } \\
\text { és }\end{array}$ & Décapodes & Potamonidae & $\begin{array}{c}\text { Potamonautes } \\
\text { brincki }\end{array}$ & 4 & 0 & 1 \\
\hline Annélides & & Rhynchobd & & $\begin{array}{c}\text { Glossiphonia } \\
\text { sp. }\end{array}$ & 0 & 0 & 5 \\
\hline & Achètes & ellida & $\begin{array}{c}\text { Glossiphoniid } \\
\text { ae }\end{array}$ & $\begin{array}{l}\text { Haementeria } \\
\text { costata }\end{array}$ & 0 & 0 & 13 \\
\hline & Oligoch & $\begin{array}{c}\text { Haplotaxid } \\
\text { a } \\
\end{array}$ & Tubificidae & $\begin{array}{c}\text { Branchiura } \\
\text { sp. }\end{array}$ & 2 & 3 & 2 \\
\hline & ètes & Lumbricida & Lumbricidae & nd & 0 & 0 & 6 \\
\hline Mollusques & $\begin{array}{c}\text { Gastéro } \\
\text { podes }\end{array}$ & $\begin{array}{l}\text { Basommat } \\
\text { ophore }\end{array}$ & Planorbidae & $\begin{array}{c}\text { Biomphalaria } \\
\text { pfeifferi }\end{array}$ & 0 & 0 & 47 \\
\hline & & & & $\begin{array}{c}\text { Biomphalaria } \\
\text { sp. }\end{array}$ & 0 & 0 & 47 \\
\hline & & & & $\begin{array}{c}\text { Planorbalus } \\
\text { sp. }\end{array}$ & 0 & 0 & 4 \\
\hline & & & Physidae & Physa acuta & 0 & 0 & 24 \\
\hline $\begin{array}{c}\text { Plathelminth } \\
\text { es }\end{array}$ & $\begin{array}{c}\text { Turbella } \\
\text { riés }\end{array}$ & Tricladida & $\begin{array}{c}\text { Dendrocoelida } \\
\mathrm{e} \\
\end{array}$ & $\begin{array}{c}\text { Dendrocoelum } \\
s p .\end{array}$ & 0 & 0 & 1 \\
\hline Total & & & & & 37 & 45 & 44 \\
\hline
\end{tabular}


La richesse taxonomique totale ainsi que La richesse taxonomique des Diptères ont été plus élevées à la station TB3 (tableau 3). La station TB2 a enrégistrée les valeurs les plus élevée de la richesse des ETO et de la richesse taxonomique des insectes. L'abondance relative des Ephéméroptères-Trichoptère-Odonate $(\% \mathrm{ETO})$ a été également fortement élevée à la station TB3.

L'indice de diversité $H$ prend des valeurs entre 0 et 5, un indice inférieur à 2 reflète un déséquilibre du milieu, par contre, des valeurs supérieures à 2 correspondent à une communauté plutôt stable. Les valeurs de l'indice de diversité de Shannon et Weaver ont oscillé entre 3,83 (station TB1) et 4,53 (station TB2). Ce qui montre que les 3 stations étudiées sont très diversifiées en macroinvertébrés benthiques. Les valeurs de l'indice d'équitabilité de Piélou ont varié de 0,71 (station TB3) à 0,83 (station TB2).

Tableau 3 : Valeurs des métriques permettant de décrire la structure des communautés des macroinvertébrés benthiques récolté durant toute la période de l'étude

\begin{tabular}{|c|c|c|c|}
\hline \multirow[b]{2}{*}{ Métriques } & \multicolumn{3}{|c|}{ Stations } \\
\hline & TB1 & TB2 & TB3 \\
\hline RTT & 37,00 & 45,00 & 44,00 \\
\hline RTD & 2,00 & 4,00 & 9,00 \\
\hline ETO & 21,00 & 25,00 & 19,00 \\
\hline RTI & 35,00 & 14,00 & 34,00 \\
\hline$\%$ ЕTO & 36,25 & 50,31 & 67,53 \\
\hline $\mathrm{H}^{6}$ & 3,83 & 4,53 & 3,89 \\
\hline $\mathrm{E}$ & 0,73 & 0,83 & 0,71 \\
\hline
\end{tabular}

RTT $=$ Richesse taxonomique totale, $\mathrm{RTD}=$ Richesse taxonomique des Diptères, ETO $=$

Richesse des Ephéméroptère-Trichoptère-Odonate, RTI = Richesse taxonomique des Insectes, $\% \mathrm{ETO}=$ Abondance relative des Ephéméroptères-Trichoptère-Odonate, $\mathrm{H}^{\prime}=$

Diversité de Shannon et Weaver, E = Equitabilité de Pielou

La Corrélation de Pearson a permis de mettre en évidence une certaine relation entre la qualité des eaux et les métriques permettant de décrire la structure des communautés de macroinvertébrés benthiques (Tableau 4). La richesse taxonomique totale, la richesse des Diptères, l'abondance relative des Ephéméroptères-Trichoptères-Odonates sont positivement et significativement corrélées avec la température, la conductivité, le taux de solides dissous et la salinité. Une corrélation positive et significative a été également observée entre la richesse taxonomique des Diptères, l'abondance relative des Ephéméroptères-Trichoptères-Odonates et la conductivité. Le même constat a été noté entre ces métriques et la salinité. Contrairement aux autres métriques, la richesse taxonomique des Ephéméroptères-Trichoptères-Odonates, la richesse taxonomique des insectes, et l'équitabilité ont montré une corrélation négative et significative avec la température, la conductivité, le taux de solides dissous et la salinité. 
La diversité n'a présenté aucune corrélation avec les différents paramètres physico-chimiques.

Tableau 4 : Valeurs de coefficients de corrélation de Pearson entre les paramètres physicochimiques et les différentes métriques des macroinvertébrés benthiques

\begin{tabular}{cccccccc}
\hline & RTT & RTD & ETO & RTI & \%ETO & H'$^{\prime}$ & E \\
\cline { 2 - 7 } pH & $-0,121$ & $-0,263$ & 0,195 & 0,149 & $-0,248$ & 0,103 & 0,264 \\
Température & $0,375^{*}$ & $0,708^{* *}$ & $-0,467^{* *}$ & $-0,339^{*}$ & $0,678^{* *}$ & $-0,212$ & $-0,682^{* *}$ \\
Conductivité & 0,307 & $0,691^{* *}$ & $-0,521^{* *}$ & $-0,404^{*}$ & $0,648^{* *}$ & $-0,284$ & $-0,699^{* *}$ \\
TDS & $0,332^{*}$ & $0,739^{* *}$ & $-0,553^{* *}$ & $-0,427^{* *}$ & $0,694^{* *}$ & $-0,298$ & $-0,746^{* *}$ \\
Salinité & 0,294 & $0,634^{* *}$ & $-0,464^{* *}$ & $-0,355^{*}$ & $0,597^{* *}$ & $-0,244$ & $-0,634^{* *}$ \\
Nitrates & 0,115 & $-0,140$ & 0,298 & 0,297 & $-0,089$ & 0,284 & 0,239 \\
$\quad$ Azote & 0,181 & $-0,044$ & 0,250 & 0,268 & 0,007 & 0,274 & 0,154 \\
ammoniacal & & & & & & & \\
Turbidité & 0,044 & $-0,035$ & 0,091 & 0,093 & $-0,019$ & 0,091 & 0,068 \\
$\quad$ MES & $-0,044$ & $-0,089$ & 0,062 & 0,046 & $-0,084$ & 0,031 & 0,087 \\
Couleur & 0,135 & 0,157 & $-0,047$ & $-0,012$ & 0,162 & 0,019 & $-0,123$ \\
Orthophosphat & 0,045 & 0,034 & 0,007 & 0,016 & 0,039 & 0,024 & $-0,018$ \\
$\quad$ es & & & & & & & \\
\hline
\end{tabular}

** Corrélation significative à $0.01, *$ Corrélation significative à 0.05

$\mathrm{RTT}=$ Richesse taxonomique totale, $\mathrm{RTD}=$ Richesse taxonomique des Diptères, $\mathrm{ETO}=$

Richesse des Ephéméroptère-Trichoptère-Odonate, RTI = Richesse taxonomique des Insectes, $\% \mathrm{ETO}=$ Abondance relative des Ephéméroptères-Trichoptère-Odonate, $\mathrm{H}^{\prime}=$

Diversité de Shannon et Weaver, E = Equitabilité de Pielou

\section{Discussions}

\section{Caractéristiques physicochimiques}

Les faibles valeurs des différents paramètres physicochimiques enregistrées pendant toute la période de l'étude sont similaires à celles obtenues dans les cours d'eau périurbains du Cameroun par Tchakonté (2016), Foto et al. (2012, 2013). Ces faibles valeurs ne traduiraient pas une absence de pollution mais plutôt un impact moindre de l'activité agricole pratiquée dans cette zone sur la qualité des eaux. L'existence d'un gradient généralement croissant de l'amont vers l'aval pour les paramètres de la température, conductivité, TDS ainsi qu'un pic de nitrate $(2,4 \mathrm{mg} / \mathrm{l})$ au niveau de la station TB2 résulteraient d'une part d'une diminution progressive du taux de couverture de la canopée vers le milieu urbain et d'autre part par l'enrichissement du cours d'eau par les ions d'origine agricole. Tchakonté (2016) a montré que les valeurs de ces variables physicochimiques subissent une nette augmentation dans les cours situés en milieu urbain contrairement au cours d'eau périurbain.

\section{Caractéristiques biologiques}

La richesse taxonomique des macroinvertébrés enregistrée dans le cours d'eau periurbain de la ville de Dschang est relativement inférieure (42 
familles) à celle obtenue par Tchakonté (2016) dans le cours d'eau Nsapé localisé dans la zone forestière relativement peu anthropisée et à l'abri de toutes perturbations. La baisse de la richesse taxonomique notée dans le cours d'eau de la région périurbaine de la ville de Dschang serait liée aux activités agricoles. En effet, l'augmentation des pratiques agricoles dans les bassins versants favorise l'homogénéisation des communautés de macroinvertébrés (Maloney et al., 2011). La quasi-totalité des peuplements des macroinvertébrés de la région périurbaine de la ville de Dschang appartient à l'embranchement des arthropodes et à la classe des insectes. Cette structure faunique est semblable à celle dressée par certains auteurs (Tchakonté, 2016; Nyamsi et al., 2014; Foto et al., 2011; Diomandé et al., 2009; Camara, 2013; Onana et al., 2016;). L'ordre des Odonates est dominant avec 8 familles et 27 taxons. Cette observation s'apparente à celle des autres auteurs où les Odonates ont été notés parmi les ordres les plus diversifiés (Karrouch et Chahlaoui, 2009; Tchakonté, 2016). Un seul taxon de crustacé a été inventorié. Ce resultat corrobore celui de Tchakonté (2016) qui a trouvé une seule espèce de crabe dans les cours d'eau Nsapé. La faible représentativité des mollusques et des Annélides qui sont des taxons polluorésistants résulterait de l'impact moindre des activités agricoles périurbaines sur la qualité des eaux, ce qui corrobore les résultats des analyses physicochimiques. Foto et al. (2011) ont notés l'emergence de ces deux taxons dans les milieux urbains fortement anthropisés. Le nombre d'individus récoltés (1833 individus) dans le ruisseau de la région agricole périurbaine de Dschang est largement inférieur à celui recensé (2553 individus) par Foto et al. (2013) dans un ruisseau périurbain au Cameroun. Ceci s'expliquerait par la forte homogénéisation des sites en zone agricole due à des stress ou des aménagements anthropiques. Les stations TB2 et TB3 représentent les stations les plus riches en espèces avec les valeurs de la richesse des ETO, des Insectes, de la diversité de Shannon et Weaver et de l'équitabilité élevées. Elles sont situées en aval des perturbations anthropiques. Ces résultats sont contraires à ceux de nombreuses études qui ont révélé que les stations localisées en aval des activités anthropiques présentent plutôt des faibles valeurs de la richesse taxonomique et de la diversité de Shannon et Weaver (Tchakonté et al., 2016; Karrouch et Chhlaoui, 2009; Tshijik et al. 2016, Onana et al., 2016, Khalaf et al., 2009). La faible vitesse du courant au niveau de la station TB2 favoriserait l'installation de certains taxons. La localisation de la station TB3 en aval direct du lac municipal de Dschang justifierait la forte diversité de macroinvertébrés observée au niveau de cette station. En effet, les lacs supportent une grande diversité d'invertébrés à cause de la présence des herbiers (Scheffer et al., 2006). De plus, les travaux de Hawkins et al. (2014) ont montré que certaines perturbations environnementales augmentent 
l'hétérogénéité biologique et la diversité. La richesse spécifique est d'autant plus élevée que le biotope est hétérogène (Ben Moussa et al., 2014). Selon Onana et al. (2016), les indices de diversités, la richesse taxonomique, l'abondance totale et relative des insectes augmentent dans les stations qui reçoivent les effluents domestiques.

\section{Conclusion}

L'utilisation des analyses des paramètres physico-chimiques des eaux et la détermination de quelques métriques des macroinvertébrés benthiques ont permis de caractériser l'état de pollution du cours d'eau Menoua traversant la zone périurbaine de Dschang. L'activité agricole a peu influencé la qualité physico-chimique de l'eau. Dans les stations en aval, elle a entrainé plutôt une diversification d'habitats et la disponibilité des ressources favorisant ainsi l'augmentation de la richesse taxonomique, de la diversité et de l'abondance des macroinvertébrés benthiques.

\section{References:}

1. Ajeagah G.A., Kekeunou S., Njiawouo Pountigni E.N. \& Foto M.S. (2014). Morphologie et abondance des stades de développement d'Ilyocoris cimicoïdes Linné 1758 (Heteroptera : Naucoridea) dans un lac) anthropisé en zone tropicale (Cameroun). Journal of Applied Bioscience, $n^{\circ} 79$, pp. $6926-6937$.

2. Babour M.T., Gerritsen J., Synder B.D. \& Stribling J.B. (1999). Rapid biossessment protols for use in stream and wadeable rivers : periphyton, benthic macroinvertebrates and fish. $2^{\text {nd }}$ edition, U.S. Environmental Protection Agency, office of water, Washington, D.C., EPA 841 - B - 99-002. 11 chapiters and appendices, 664p.

3. Ben Moussa A., CHahlaoui A., Rour E., Chahboune M. (2014). Diversité taxonomique et structure de la macrofaune benthique des eaux superficielles de l'oued khoumane. Moulay idriss Zerhoun, Maroc. J. Mater. Environ, pp. 183-198.

4. Brown D.S. (1994). Freshwater Snails of Africa and their Medical Importance. CRC Press, Taylor \& Francis Ltd. Ed, London, $2^{\text {nd }}$ revised edition, 1994, 609p.

5. Camara I.A. (2013). Composition, structure et déterminisme des macroinvertébrés de la rivière Banco (Parc National du Banco, Côte d'Ivoire). Thèse de Doctorat, Université Nangui Abrogoua, 151p.

6. Cisse I., Tandia A.A., Fall T.S., Diop S.H.E. (2003). Usage incontrôlé des pesticides en agriculture périurbaine : cas de la zone des Niayes au Sénégal. Cahiers Agriculture, pp. 181-6.Day J.A., Harrison A.D. \& De Moor I.J. (2002). Guides to the Freshwater Invertebrates of Southern Africa, Volume 9: Diptera. Water 
Research Commission Report, No. TT 201/02, Pretoria-South Africa, 210 .

7. Day J.A., Stewart B.A., De Moor I.J. \& Louw A.E. (2001). Guides to the Freshwater Invertebrates of Southern Africa, Volume 4: Crustacea III. Water Research Commission Report, No. TT 141/101, Pretoria-South Africa, 151p.

8. Dejoux C., Elouard J.M., Forge P. \& Maslin J.L. (1981). Catalogue iconographique des insectes aquatiques de Côte d'Ivoire. ORSTOM 42, $178 \mathrm{p}$.

9. De Moor I.J. \& Day J.A. (2002). Guides to the Freshwater Invertebrates of Southern Africa, Volume 6 : Arachnida \& Mollusca. Chapter 3 : Mollusca. Water Research Commission Report, No. TT 182/02, Pretoria-South Africa, pp. 42-125.

10. De Moor I.J., Day J.A. \& De Moor F.C. (2003). Guides to the Freshwater Invertebrates of Southern Africa, Volume 7 : Insecta I. Ephemeroptera, Odonata \& Plecoptera. Water Research Commission Report, No. TT 207/03, Pretoria-South Africa, 301p.

11. Diomande D., Bony Y.K., Edia O.E., Konnan K.F., Gourene G. (2009) Diversité des Macroinvertébrés benthiques de la rivière Agnéby (Côte d'Ivoire; Afrique de l'ouest). European journal of Scientific Research, $n^{\circ} 35$, pp. 328-377.

12. Diomande D., Gourene G., Sankare Y. \& Zabi S.G. (2000). Synopsis de la classification des larves et des nymphes de Diptères Chironomidea des écosystèmes dulçaquicoles de l'Afrique de l'Ouest. Clés de détermination des sous-familles, des tribus et des genres. Archives scientifiques $d u$ Centre de Recherches Océanologiques d'Abidjan, $n^{\circ} 17$, pp.1-31.

13. Durand J.R. \& Leveque C. (1981). Flore et faune aquatique de l'Afrique Sahélo-soudanenne. Tome II. Edition de L'ORSTOM, Paris, 517p.

14. Foto M.S., Koji E., Ajeagah G.A., Bilong Bilong C.F. \& Njine T. (2012). Impact of dam construction on the diversity of benthic macroinvertebrates community in a periurbain stream in Cameroon. The International Journal of Biotscience, $n^{\circ}$ 2, pp. $137-145$.

15. Foto M.S., Tchakonte S., Ajeagah G.A., Zebaze T.S.H., Bilong Bilong C.F. \& Njine T. (2013). Water quality assessment using benthic macroinvertebrates in a periurban sream (Cameroon). The International Journal of Biotechnology, $n^{\circ} 2$, pp. $91-104$.

16. Foto M.S., Zebaze T.S.H., Nyamsi T.N.L., Ajeagah G.A., Njine T. (2011). Evolution Spatiale de la diversité des Peuplements de Macroinvertébrés Benthiques dans un cours d'eau Anthropisé en 
Milieu Tropical (Cameroun). European Journal of Scientific Research, $n^{\circ}$ 55, pp. 291-300.

17. Hawkins C.P., Mykrä H., Oksanen J. and Vander Laan J.J. (2014). Environmental disturbance can increase beta diversity of stream macroinvertebrate assemblages. Global Ecology and Biogeography, $n^{\circ} 24, p p$. 483-494.

18. INS (2007). Institut National de Statistique, Yaoundé, Cameroun. "Annuaire statistique du Cameroun ».

19. Karrouch L., Chahlaoui A. (2009). Bio-evaluation de la qualite des eaux de l'Oued Boufekrane (Meknes, Maroc). Biomatec Echo, $n^{\circ}$, pp. 6-17.

20. Khalaf G., Slim K., Abi-Ghanem C., Nakhle K. et Fakhri M. (2009). Caractérisation et corrélations des paramètres biotiques et abiotiques des eaux du Nahr El Bared. Lebanese Science Journal, $n^{\circ} 1$, pp. 3-21.

21. Kouam K.G.R., Rosillon F., Mpakam H.G. et Nono A. (2010). Enjeux sanitaires, socio-économiques et environnementaux liés à la réutilisation des eaux usées dans le maraîchage urbaine à Yaoundé au Cameroun: cas du bassin versant de l'Abiergué. Colloque Eau, Déchets et Développement Durable, Alexandrie, Egypte, 28 - 31 mars, 8p.

22. Leigh C., Burford M.A., Roberts D.T., Udy J.W. (2010). Predicting the vulnerability of reservoirs to poor water quality and cyanobacterial blooms. Water Research, $n^{\circ}$ 44, pp. 4487-4496.

23. Maloney K., Munguia P. and Mitchell R. M. (2011). Anthropogenic disturbance and landscape patterns affect diversity patterns of aquatic benthic macroinvertebrates. Journal of the North American Benthological Society, $\mathrm{n}^{\circ}$ 30, pp. 284-295.

24. Margiotta M. (1997). Agriculture périurbaine et Système d'Approvisionnement et de Distribution Alimentaire (SADA). Programme FAO «Approvisionnement et distribution alimentaires des villes $\gg$.

25. Moisan J., Pelletier L. (2008). Guide de Surveillance Biologique Basée sur les Macroinvertébrés Benthiques d'Eau Douce du Québec - Cours d'Eau Peu Profonds à Substrat Grossier. Direction du Suivi de l'Etat de l'Environnement, Ministère du Développement Durable, de l'Environnement et des Parcs: Québec.

26. Nyamsi T.N.L., Foto M.S., Zebaze T.S.H., Onana F.M., Adandedjan D., Tchakonte S., Yemele T.C., Koji E. \& Njine T. (2014). Indice Multimétrique des Macroinvertébrés Benthiques Yaoundéens (IMMY) pour l'évaluation biologique de la qualité des eaux de cours d'eau de la Région du Centre Sud Forestier du Cameroun. European Journal of Scientific Research, $n^{\circ}$ 123, pp. 412-430. 
27. Onana F.M., Zebaze T.S.H., Koji E., Nyamsi T.N.L. et Tchakonte S. (2016). Influence of municipal and industrial pollution on the diversity and the structure of benthic macro-invertebrates community of an urban river in Douala, Cameroon. Journal of Biodiversity and Environmental Sciences (JBES), $n^{\circ}$ 8, pp. 120-133.

28. Rodier J., Legube B., MerleT N. (2009). L'analyse de l'eau. Paris, Dunod, 9e édition, $1579 \mathrm{p}$.

29. Rosenberg D.M. \& Resh V. H. (1993). Freshwater biomonitoring and benthic macroinvertebrates. Chapman and Hall, London, 1993.

30. Sass L.L., Bozek M.A., Hauxwell J.A., Wagner K., Knight S. (2010). Response of aquatic macrophytes to human land use perturbations in the watersheds of Wisconsin lakes, U.S.A. Aquatic Botany, $n^{\circ}$ 93, $p p$. 1-8.

31. Scheffer M.G.J., Van Geest K., Zimmerm E, Jeppesen M., Sondergaard M. G., Butlerhanson M. A., Declerck S. and De Meester L. (2006). Small habitat size and isolation can promote species richness: second-order effects on biodiversity in shallow lakes and ponds. Oikos, $n^{\circ} 112$, pp. 227-231.

32. Stals R. \& De Moor I.J. (2007). Guides to the Freshwater Invertebrates of Southern Africa, Volume 10 : Coleoptera. Water Research Commission Report, No. TT 320/07, Pretoria-south Africa, $275 p$.

33. Stark J.D., Boothroyd K.G., Harding J.S., Maxted J.R. \& Scarsbrook M.R. (2001). Protocols for Sampling Macroinvertebrates in Wadeable Streams. New Zealand Macroinvertebrates working group, report no.1, Ministry for the Environment and sustainable Management, fund project no.5103, 57p.

34. Tachet H., Richoux P., Bournaud M. \& Usseglio-Polatera P. (2010). Invertébrés d'eau douce : systématique, biologie et écologie. CNRS édition, Paris, 588p.

35. Tchakonte S. (2016). Diversité et structure des peuplements de macroinvertébrés benthiques des cours d'eau urbains et périurbains de Douala (Cameroun). Thèse de Doctorat/PhD. Université de Yaoundé, 200p.

36. Temgoua E., Ntangmo Tsafack H. and Njine T. (2012). "Vegeta-ble Production Systems of Swamps Zone in Urban Envi-ronment in West Cameroon: Case of Dschang City," Universal Journal of Environmental Research and Technology, $n^{\circ} 2$, pp. 83-92.

37. Tshijik J-C. K., Akaibe D., Micha J-C. et Pwema V.K. (2016). Analyse comparative de la diversité taxonomique et de la qualité écologique de l'eau des rivières Lukunga et Mangengenge à 
Kinshasa. International Journal of Innovation and Applied Studies, $\mathrm{n}^{\circ} 16: 260-271$.

38. Wade C.S. (2003). L'utilisation des pesticides dans l'agriculture périurbaine et son impact sur l'environnement. Etude menée dans la région de Thiès, Thèse Pharmacie, Dakar, UCAD, 55 p. 